\title{
Evaluación del efecto de un programa de ejercicio físico sobre la capacidad cardiorrespiratoria en académicos de la Universidad de Sonora con síndrome metabólico: un estudio piloto
}

Evaluation of the effect of a physical exercise program on cardiorespiratory capacity in academics with metabolic syndrome at the University of Sonora: a pilot study

María Elena Chávez Valenzuela, Melanie Valdez García, Alejandrina Bautista Jacobo, Graciela Hoyos Ruiz, Nidia Carolina

Barahona Herrejón, Carlos Ernesto Ogarrio Perkins

Universidad de Sonora (México)

Resumen. El objetivo de este estudio piloto fue evaluar los efectos de un programa de intervención de ejercicio físico sobre la capacidad cardiorrespiratoria en los académicos de la Universidad de Sonora que presentaron síndrome metabólico. Se llevó a cabo un estudio preexperimental con diseño preprueba-postprueba en un solo grupo de 10 sujetos que participaron de manera voluntaria (47.10 \pm 11.10 años). El programa de intervención tuvo una duración de 13 semanas con cuatro sesiones semanales que incluyeron actividades de tipo aeróbicas como fueron la caminata, natación, bicicleta estacionaria, baile y actividades al aire libre, entre otras. Las medidas antropométricas y el VO2máx fueron tomadas al inicio y al final de la intervención. Se utilizó la prueba t de muestras dependientes $(p<.05)$ y en otros casos la prueba deWilcoxon dado el incumplimiento de normalidad. El programa logró un aumento significativo en la variable principalVO2máx de $10.41(\mathrm{ml} / \mathrm{kg} / \mathrm{min})(p=.002)$, el índice de masa corporal se redujo $.94 \mathrm{~kg} / \mathrm{m}^{2}(p=.004)$, la masa grasa en $2.40 \mathrm{~kg}(p=.014)$, los niveles de triglicéridos en $14.41 \mathrm{mg} / \mathrm{dL}$ y la circunferencia de cintura $3.98 \mathrm{~cm}(p=.016)$. La presión arterial sistólica y diastólica también disminuyeron al término del programa, pero estos cambios no fueron estadísticamente significativos $(p>.05)$. Este trabajo se focalizó en los padecimientos de síndrome metabólico, encontrando mejorías principalmente en la capacidad cardiorrespiratoria. Es importante destacar que, en México este tipo de intervenciones son escasas, por lo que se sugiere seguir investigando al respecto y documentar los hallazgos.

Palabras clave: síndrome metabólico, capacidad cardiorrespiratoria, masa grasa, ejercicio físico, académicos.

\begin{abstract}
The purpose of this pilot study was to evaluate the effects of a physical exercise intervention program on cardiorespiratory capacity in academics from the University of Sonora who manifested metabolic syndrome. A pre-experimental study with a pre and post test design was carried out in a single group of 10 individuals who participated voluntarily ( $47.10 \pm 11.10$ years). The intervention program lasted 13 weeks with four weekly sessions that included aerobic activities such as walking, swimming, stationary cycling, dancing, and outdoor activities. Anthropometric measurements andVO2max were taken at the beginning and end of the intervention. The dependent samples t-test was used $(p<.05)$, and in other cases, theWilcoxon test in case of the non-compliance with normality. The program achieved a significant increase in the main variableVO2max of $10.41(\mathrm{ml} / \mathrm{kg} / \mathrm{min})(p=.002)$, the body mass index was reduced by $0.94 \mathrm{~kg} / \mathrm{m}^{2}(p=.004)$, the fat mass by 2.40 $\mathrm{kg}(p=.014)$, triglyceride levels at $14.41 \mathrm{mg} / \mathrm{dL}$ and waist circumference $3.98 \mathrm{~cm}(\mathrm{p}=.016)$. Systolic and diastolic blood pressure also decreased at the end of the program, but these changes were not statistically significant ( $\mathrm{p}>.05$ ). This work focused on metabolic syndrome disorders, finding improvements mainly in cardiorespiratory capacity. It is important to highlight that this type of intervention in Mexico is scarce, so it is suggested to continue investigating in this regard and document the findings.
\end{abstract}

Keywords: metabolic syndrome, cardiorespiratory capacity, fat mass, physical exercise, academics.

\section{Introducción}

Las enfermedades crónico-degenerativas no trasmisibles (ECNT) han tenido un nivel de prevalencia en constante aumento, sin embargo, es probable que los efectos de estas vayan disminuyendo si se reactiva el hábito de la actividad física, sobre toda en personas con síndrome metabólico (SM). Algunos estudios sobre adultos mayores sugieren que el ejercicio físico y actividades que conlleve a la pérdida de peso puede reducir el número de componentes del SM (Villareal, Miller, Banks, Fontana, Sinacore \& Klein, 2006; Yassine, Marchetti,

Fecha recepción: 21-04-20. Fecha de aceptación: 27-10-21

Alejandrina Bautista Jacobo

alejandrina.bautista@unison.mx
Krishnan, Vrobel, Gonzalez, \& Kirwan, 2009), además de garantizar una condición física funcional y riesgo de caídas (Catañeda-Lechuga, Macias-Ruvalcaba, GallegosSánchez, \& Villareal-Angeles). De acuerdo a los criterios del National Cholesterol Eduaction Program (NCEP, 2001) el síndrome metabólico se presenta con la combinación de tres o más factores de riesgo como la hipertensión, dislipidemia, resistencia a la insulina, obesidad abdominal y riego de enfermedades cardiovasculares. Además, la edad y masa corporal están directamente relacionadas con el desarrollo del SM (Ford, 2005).

Según datos de la Organización Mundial de la Salud (OMS, 2013) del 2013, las ECNT se presentaban con mayor aumento en los países de bajos ingresos y medianos alcanzando una magnitud preocupante, derivado de 
la baja actividad física y el alto consumo de alimentos en grasa. En tanto en Europa, en el mismo año, el 25\% de la población presentaba síndrome metabólico y en países como América, al menos el 25\% de los adultos eran portadores de esta afección (Ramírez, Rosety, Becerro, Rosety, Ordoñez, Rosety-Rodríguez, RodríguezPareja et al., 2012). En cuanto a México, la Encuesta Nacional de Salud y Nutrición (ENSANUT) realizada en el año 2012, dio como dato que la prevalencia combinada de sobrepeso y obesidad era de 71.2\% (Gutiérrez, Rivera, Shamah, Oropeza \& Hernández, 2012).Al 2016, esta prevalencia creció a 72.5\% para la población adulta mexicana (ENSANUT MC, 2016). Para el Estado de Sonora, México, según la ENSANUT (2016) la prevalencia de sobrepeso y obesidad en hombres estaba en $70.6 \%$ y $76.69 \%$ en mujeres.

En cuanto a las enfermedades cardiovasculares, éstas tienen comorbilidad asociados a otros padecimientos. Las variables de riesgo de estas necesitan ser tratadas por diversos medios funcionales para el tratamiento y disminución de la prevalencia de los efectos de las enfermedades crónico-degenerativas. El impacto del problema de salud-enfermedad que se genera en las personas con patologías cardiovasculares, denota que las medidas tradicionales encaminadas a incidir de manera positiva en el control de dichas enfermedades no han arrojado resultados satisfactorios (Quintana-Zavala, Vílchez-Barboza, Figueroa-Ibarra, García-Puga, Tinajero-González \& Salazar-Ruibal, 2018). Debido a estas premisas es necesario abordar los tratamientos utilizando metodologías de intervención efectivas y distintas.

En un estudio realizado a trabajadores universitarios del Estado de México para asociar el estilo de vida con riesgo cardiovascular, se obtuvo como resultado que la actividad física moderada-vigorosa es un factor del estilo de vida asociado con menor riesgo cardiovascular (Cerecero, Hernández, Aguirre, Valdés \& Huitrón, 2009). Desde reflexiones analizadas por medio de revisiones sistemáticas sobre los beneficios de la actividad física, Alvarez-Pitti, Casajús, Leis, López de Lara, Moreno \& Rodríguez (2020), mencionan que la actividad física funciona como un tratamiento medicinal para las enfermedades crónico degenerativas no trasmisibles.

En cuanto a estudios con intervenciones de la actividad física para analizar la asociación del consumo máximo de oxígeno con la actividad física y el sedentarismo en el síndrome metabólico, se detectó que los sujetos con SM con el factor sobrepeso u obesidad que afirmaron cumplir con las recomendaciones sobre actividad física, alcanzaron mayor consumo máximo de oxígeno, mientras que los que llevaban actividades sedentarias no lograron cambios en el VO2máx. (Tojal, AlonsoGómez, Alberich, Wärnberg, Sorto, Portillo, Schroder et al., 2020). La Actividad física, aparte de ser un factor protector de las ECNT, también está documentado que se relaciona con la mejora de variables como la ansiedad y depresión (Guillen, Bueno, Gutiérrez \& Guerra, 2018; Chávez, Bautista, García, Fuentes, Ogarrio, Montaño del Cid \& Hoyos, 2018), el estado de ánimo (Villareal, Moncada, Gallegos \& Ruiz Juan, 2016), la autoestima y la calidad de vida (García \& Froment, 2018).

Observando los múltiples padecimientos de la actualidad, debidas en su mayoría al sedentarismo y alimentos hipercalóricos, una actividad que aporta beneficios sin riesgos de lesionarse es la caminata. Cuando esta se lleva a cabo de manera adecuada y constante, puede prevenir el aumento de peso, además de tener menos impacto en las articulaciones. Aunado a esto, aumenta el gasto calórico y tiene un gran valor para incentivar a los individuos para incorporarse a la práctica de ejercicio físico.

La OMS recomienda a las personas adultas caminar al menos 10,000 pasos al día para mantenerse en forma. $\mathrm{Al}$ caminar se ponen en movimiento un 30\% de los músculos del cuerpo que es una forma segura no solo para mantenernos en forma, sino también para combatir con problemas cardiovasculares, sobrepeso u osteoporosis. Otros de los beneficios es el fortalecimiento de los músculos de la cadera, muslos y piernas; regulación de la presión sanguínea; y reducción del estrés. Autores enfatizan que, una caminata entre 3,000 y 4,000 pasos a ritmo rápido supone un gasto aproximado de 150 calorías (Abellán, Sainz de Baranda, Ortín, Saucedo, Gómez \& Leal, 2014).

Existen gran cantidad de estudios de análisis y propuestas de intervención, sin embargo, es necesario emplear intervenciones con seguimientos en tiempos prolongados. Justamente, la metodología empleada funge como un estudio propositivo a futuras investigaciones con resultados cuantitativos que devienen de un seguimiento asiduo. El objetivo del presente estudio piloto fue: evaluar los efectos del programa de intervención de ejercicio físico sobre la capacidad cardiorrespiratoria en los académicos de la Universidad de Sonora que presentaban SM.

Partiendo del hecho de que el ámbito de la docencia influye de manera significativa en la educación de los futuros profesionistas, la salud de los académicos desa- 
rrolla un papel importante. La presencia de enfermedades no transmisibles para cierta población de académicos de la Universidad de Sonora, impiden en ocasiones cumplir con sus funciones. También se convierten en incapacidades y suplencias, lo cual genera gastos tanto a la institución como al presupuesto de salud pública y del propio bolsillo. En concordancia con el objetivo general del estudio, se plantea la siguiente pregunta de investigación:

¿Un programa de intervención de ejercicio físico tendrá un efecto significativo en la capacidad cardiorrespiratoria de los académicos de la Universidad de Sonora que presenten síndrome metabólico?

\section{Material y método}

\section{Participantes}

Se llevó cabo un estudio pre-experimental con diseño preprueba-postprueba en un solo grupo, basado en la propuesta metodológica de Hernández, Fernández \& Baptista (2010), en una muestra no probabilística por conveniencia de 10 académicos de la Universidad de Sonora. El 70\% eran del sexo femenino y 30\% del masculino y cuyo rango de edad fue de 33 a 60 años (47.10 $\pm 11.10)$. En la Tabla 1, se muestran las características de los participantes del estudio al inicio del programa. Los criterios de inclusión para los participantes fueron: ser académicos de la Universidad de Sonora, tener una edad mínima de 30 años y máxima de 65 y presentar al menos tres o más componentes del síndrome metabólico (triglicéridos >150 mg/dl, nivel de colesterol HDL < $40 \mathrm{mg} / \mathrm{dl}$ en varones $\mathrm{y}<50 \mathrm{mg} / \mathrm{dl}$ en mujeres, una presión arterial sistólica e»130 mmHg o diastólica e» $85 \mathrm{mmHg}$, un nivel de glucosa en plasma en ayunas e»100 mg/dl); circunferencia de cintura en mujeres de $>80 \mathrm{~cm}$ y en hombres $>90 \mathrm{~cm}$ (Federación Internacional de Diabetes, 2006). Además, presentar constancia médica y firmar la carta de consentimiento informado.

\begin{tabular}{lcc}
$\begin{array}{l}\text { Tabla } 1 \\
\text { Datos generales de los participantes }\end{array}$ & $\mathrm{N}$ & \\
\hline Variables & 7 & 70.0 \\
\hline Sexo & 3 & 30.0 \\
Femenino & & \\
Masculino & 2 & 20.0 \\
Clasificación según la presiona arterial & 3 & 30.0 \\
Crónica & 1 & 10.0 \\
Fronteriza & 4 & 40.0 \\
Normal & & \\
Optima & 1 & 10.0 \\
Clasificación según el IMC & 6 & 60.0 \\
Sobrepeso grado II & 2 & 20.00 \\
Obesidad tipo I & 1 & 10.00 \\
Obesidad tipo II & & \\
Obesidad tipo 3 & 5 & 50.0 \\
Clasificación según los niveles de colesterol & 4 & 40.0 \\
Normal & 1 & 10.0 \\
Riesgo & & \\
Muy elevado & 8 & 80.0 \\
Clasificación según los niveles de triglicéridos & 2 & 20.0 \\
Normal & & \\
Alto &
\end{tabular}

Los criterios de exclusión: personas que no presentaron SM, que presentaran alguna patología que les impedía realizar algún tipo de actividad física, que fuesen menores a la edad establecida y por consecuencia mayor a la misma. Además, que no contaran con autorización médica y no hayan firmado el consentimiento informado. Como criterio de eliminación: si presentaban alguna lesión, alguna consecuencia negativa derivada de la actividad física o que deseaban salir por voluntad propia. Los participantes voluntarios fueron citados a reunión general, donde se les brindo información sobre los objetivos de la investigación, los procedimientos que se llevarían a cabo durante el estudio, así como los beneficios y riesgos, mediante un consentimiento informado el cual deberían firmar en caso de estar de acuerdo. Además, se hizo énfasis en la confiabilidad de la información obtenida. Esta investigación fue revisada y avalada por el Comité de Ética en Investigación por la Universidad de Sonora y se realizó de acuerdo con los procedimientos aprobados con la declaración de Helsinki.

\section{Instrumentos y Recolección de la información}

Se utilizó el Test de Rockport (Kline, Porcari, Hintermeister, Freedson, Ward, McCarron et al., 1987), el cual está diseñado particularmente para aquellas personas que no pueden correr debido a una pobre condición cardiorrespiratoria. La prueba de Rockport solo requiere que el participante camine la distancia de una milla lo más rápido posible. La frecuencia cardiaca de los participantes debe, como mínimo, subir a 120 latidos/minuto al finalizar la prueba. Se habrá de estimar la capacidad aeróbica sobre la base de las variables edad, género y tiempo transcurrido durante la milla y la frecuencia cardiaca alcanzada al finalizar la prueba. Para estos propósitos, la prueba permite utilizar una ecuación de regresión, de manera que se pueda estimar la tolerancia aeróbica o consumo de oxígeno máximo (ml $\cdot \mathrm{kg}-1 \cdot \min -1)$.

$$
\begin{aligned}
& \text { VO2máx }(\mathrm{ml} \bullet \mathrm{kg}-1 \cdot \min -1)=132.6-(.17 \times \mathrm{MC}) \\
& -(.39 \times \text { Edad })+(6.31 \times \mathrm{G})-(3.27 \times \mathrm{T})-(.156 \times \mathrm{FC}) \\
& \text { Donde: } \\
& \mathrm{G}=\text { Género o Sexo }(0=\text { mujeres; } 1=\text { varones }) \\
& \mathrm{MC}=\text { Masa o peso corporal }(\mathrm{kg}) \\
& \mathrm{T}=\text { Tiempo transcurrido durante la prueba (minu- }
\end{aligned}
$$
tos)

$\mathrm{FC}=$ Frecuencia cardiaca o pulso palpado extrapolado a un minuto (latidos/minuto).

Tomando en cuenta que los participantes presentaban algunos factores del SM y las causas que desencadenan el mismo, se empleó la técnica de la caminata. Este 
medio es uno de los ejercicios más seguros para mejorar la capacidad cardiorrespiratoria, la cual es de bajo impacto y no lesiona las articulaciones. El instrumento utilizado para contar los pasos fue a través de la aplicación de PACER, muy práctica, sin costo, y fácil de instalar en cualquier dispositivo. Esta aplicación permitió contabilizar los pasos realizados durante el día, sumándose a los pasos logrados durante el ejercicio físico sistematizado, de acuerdo a la soportado en la Guía para la prescripción del ejercicio físico en pacientes con riesgo cardiovascular (Abellán et al., 2014, p.22).

Para conocer el porcentaje de la carga de acuerdo a las necesidades de cada persona, se utilizó el Método de Karvonen respaldada en la fórmula de Abellán et al., que consiste en restar a la FC máx. la FC medida en reposo en posición de bipedestación, obteniendo así la FCE. Este resultado se multiplica el porcentaje de la intensidad deseada del ejercicio (40\%-85\% de la FCR) y ambos valores se les vuelve a sumar la FC de reposo. Obteniendo así, los dos límites entre los que debe mantener la FC de entrenamiento:

FC esperada $=[($ FCmáx. - FC reposo $) \mathrm{x} \%$ de trabajo] + FC reposo

Para la carga de trabajo de EF se consideró el resultado obtenido de las evaluaciones iniciales de la FC, determinándose trabajar con un porcentaje de 50 a 60\% con intensidad moderada las primeras seis semanas. Después se fue realizando la progresión de la carga de acuerdo al aumento de condición física de los participantes. Este porcentaje es considerado el nivel del ritmo cardiaco para aquellas personas que están iniciando un programa de entrenamiento, que han estado inactivos por mucho tiempo, o que están en rehabilitación.

El siguiente paso fue la toma de muestra sanguínea. Los participantes asistieron en ayunas, citándose en el Laboratorio de Análisis Clínicos e Investigación de la Universidad de Sonora (LACIUS). Posteriormente, se convocó para realizar las mediciones antropométricas apoyados por el personal del Centro de Promoción a la Salud Nutricional de la misma institución. Los resultados de los análisis sanguíneos y antropométricos, fueron examinados por un médico responsable del programa de salud estudiantil de la Universidad de Sonora, quien facilitó el certificado de salud de cada participante para valorar su intervención en el programa de ejercicio físico sistematizado.

Para las medidas antropométricas, se empleó un estadiómetro de la marca SECA con capacidad hasta $220 \mathrm{~cm}$, el sujeto permaneció en posición de pie en contra del estadiómetro, los talones debían permane- cer juntos, trasero y escapulas se encontraban totalmente pegadas a éste. La cabeza debía estar posicionada en el plano de Frankfort, y el arco orbital inferior en línea recta con trago de la oreja.

Para la circunferencia de cintura el participante permaneció de pie, erguido y con el abdomen relajado, los brazos permanecieron a los costados y los pies juntos. La circunferencia de cintura se estableció midiendo la cintura a nivel del ombligo, realizándose dos tomas, cada toma se capturaba al final de una espiración normal empleando una cinta antropométrica de fibra de vidrio (GÜLICK, 0 a 150 cm). Para medir el peso en kilogramos y el porcentaje de grasa fue estimado por SECA MBCA (Medical Body Composition Analyzer, SECA Gmbh \& Co. Kg Hammer Steindamm 9-25) partiendo de la posición del participante como medida estándar, se mide con la persona en decúbito supino, permaneciendo 10 minutos antes de la medición, con los brazos separados entre 30 y $45^{\circ}$ del tronco y los muslos no se deben tocar entre sí (Heyward, 2008). Para la toma de la presión arterial se siguieron las indicaciones sugeridas por la Organización Panamericana de la Salud, donde indica que para obtener una medición precisa de la presión arterial, es necesario la preparación del paciente antes de tomar la presión arterial, acondicionar un espacio sin ruidos, no conversar, apoyar el brazo a la altura del corazón, colocar el manguito en el brazo sin ropa, apoyar los pies, no cruzar las piernas, tener la vejiga vacía y mantener apoyada la espalda. La técnica utilizada también recomendad por la OPS, es realizar las mediciones en ambos brazos, en la visita inicial, y, posteriormente, seguir usando el brazo con la medición más alta (OPS, 2021).

Para el cálculo del Índice de Masa Corporal (IMC), se empleó la clasificación de la Sociedad Española para el estudio de la Obesidad (SEEDO, 2000) donde peso insuficiente es < 18.5; Normo peso: 18.5-24.9; Sobrepeso grado I: 25-26.9; Sobrepeso grado II: 27-29.9; Obesidad tipo I: 30-34.9; Obesidad tipo II: 35-39.9; Obesidad tipo III (mórbida): 40-49.9 y Obesidad tipo IV (extrema) $>50$.

\section{Estructura del Programa Propuesto}

El Programa de ejercicio físico se apoyó en las indicaciones del Institute of Medicine (IOM) que recomienda 60 minutos de actividad física de intensidad moderada, que se relaciona para mantener un peso corporal saludable y evitar un aumento de peso nocivo para su salud. Este representa una cantidad de tiempo suficiente para personas sedentarias, a medida que van adaptándose al 
esfuerzo físico y mejorando su condición física, se aplica la progresión de la carga paulatinamente (Heyward, 2008, pp.4-5). Este se llevó a cabo durante 13 semanas, dirigido a mejorar la resistencia cardiorrespiratoria, haciendo participar los grandes grupos musculares, con un nivel de bajo impacto para no afectar las articulaciones, tomando en cuenta las características y necesidades de los participantes. En tanto los ejercicios de natación, un día a la semana de las primeras cuatro semanas, seguida de la bicicleta estacionaria, a partir de la semana cinco a la novena, trabajando 20 minutos con una intensidad moderada entre 55 a 65\% de la FCmáx. La actividad correspondiente al baile, se practicó una vez a la semana durante las últimas cinco semanas del programa, variando la intensidad según el ritmo de la música, de moderado a vigoroso (aumentando la intensidad en lapsos de segundos), midiéndose a través de la FC y del VO2máx., indicadores que permiten aumentar la intensidad y la duración. Mientras que la capacidad condicional de la fuerza fue organizada con ejercicios sin implementos, dos días a la semana con seis a ocho ejercicios, con una repetición de ocho a 12. En cuanto a la flexibilidad se dedicaron dos días a la semana, utilizando el método de Pilates de piso para principiantes, trabajando estiramientos, amplitud articular, equilibrio postural y corrección de posturas, de ocho a 10 ejercicios con ocho a 10 repeticiones. En cuanto al trabajo de respiración se empleó el método de Vinyasa yoga, este método se enfoca en sincronizar el ejercicio con la respiración, incluidos en los cuatro días de actividad a la semana, intervenía desde el calentamiento, parte medular o principal y enfriamiento (Ver Tabla 2).

\begin{tabular}{|c|c|c|c|c|c|}
\hline \multicolumn{6}{|c|}{ Programa general de entrenamiento } \\
\hline & Medios & Frecuencia & Duración & Intensidad & Observaciones \\
\hline $\begin{array}{c}\text { Resistencia } \\
\text { cardiorrespiratoria }\end{array}$ & caminata & $\begin{array}{l}3 \text { días a la } \\
\text { semana }\end{array}$ & $\begin{array}{c}\text { Entre } 20 \text { y } 25 \\
\text { minutos }\end{array}$ & $\begin{array}{c}\text { Moderada } \\
\text { De } 50 \% \text { al } 70 \%\end{array}$ & $\begin{array}{l}\text { El tiempo dependía de la capacidad física del } \\
\text { participante que oscilaba entre } 20 \text { a } \\
\text { 25minutos y la intensidad fue progresando de } \\
\text { acuerdo a la mejora de la FC }\end{array}$ \\
\hline $\begin{array}{c}\text { Resistencia } \\
\text { cardiorrespiratoria }\end{array}$ & Natación & $\begin{array}{l}1 \text { día a la } \\
\text { semana }\end{array}$ & $\begin{array}{c}4 \text { semanas (30 } \\
\text { min) }\end{array}$ & Baja a moderada & Se consideró para las primeras 4 semanas \\
\hline $\begin{array}{c}\text { Resistencia } \\
\text { cardiorrespiratoria }\end{array}$ & $\begin{array}{l}\text { Bicicleta } \\
\text { estacionaria }\end{array}$ & $\begin{array}{l}1 \text { día a la } \\
\text { semana }\end{array}$ & $\begin{array}{l}\text { Por } 4 \text { semanas } \\
\quad(20 \mathrm{~min})\end{array}$ & $\begin{array}{c}\text { Moderada } \\
\text { Entre } 55 \text { a } 65 \% \\
\text { de la FCmáx }\end{array}$ & Se consideró para las 4 semanas intermedias \\
\hline $\begin{array}{c}\text { Resistencia } \\
\text { cardiorrespiratoria }\end{array}$ & baile & $\begin{array}{l}1 \text { día a la } \\
\text { semana }\end{array}$ & $\begin{array}{l}\text { Por } 5 \text { semanas } 1 \\
\quad(30 \mathrm{~min})\end{array}$ & $\begin{array}{l}\text { Moderada de } 55 \text { a } \\
65 \% \text { FCmáx }\end{array}$ & Se consideraron las 5 últimas semanas \\
\hline Fuerza & $\begin{array}{c}\text { Sin } \\
\text { implementos }\end{array}$ & $\begin{array}{l}2 \text { días a la } \\
\text { semana }\end{array}$ & cicios & $\begin{array}{c}8 \text { a } 12 \\
\text { repeticiones }\end{array}$ & \\
\hline Flexibilidad & $\begin{array}{l}\text { Pilates de } \\
\text { piso }\end{array}$ & $\begin{array}{c}2 \text { días a la } \\
\text { semana }\end{array}$ & $\begin{array}{l}\text { De } 8 \text { a } 10 \\
\text { ejercicios }\end{array}$ & $\begin{array}{l}\text { De } 8 \text { a } 10 \\
\text { repeticiones }\end{array}$ & Sin presentar dolor \\
\hline $\begin{array}{l}\text { Sesiones de trabajo } \\
\text { de la respiración }\end{array}$ & IvasaYoga & $\begin{array}{l}4 \text { días a la } \\
\text { semana }\end{array}$ & $\begin{array}{l}\text { Durante toda } \\
\text { la sesión }\end{array}$ & $\begin{array}{l}\text { Inicio, durante y } \\
\text { final de la sesión }\end{array}$ & $\begin{array}{l}\text { Este medio ayuda a aprender a sincronizar la } \\
\text { respiración con cada movimiento corporal }\end{array}$ \\
\hline
\end{tabular}

Para el cálculo de la carga de trabajo se tomaron de referencia los resultados del nivel inicial, factores y componentes del síndrome metabólico. Fue un programa de entrenamiento continuo, donde las primeras cuatro semanas se trabajó con un porcentaje inicial del 50 al $60 \%$ de la FCmáx, dado su condición de que cada volun- tario presentaba sobre peso, obesidad y bajo nivel de actividad física, causantes del síndrome metabólico. Debido al progreso obtenido, en las últimas 9 semanas se incrementó progresivamente la carga de un 55 a 70\% con intensidad moderada de larga duración considerando en cada sesión el protocolo de revisión (la toma de la presión arterial, glucosa en sangre, frecuencia cardiaca, calentamiento y enfriamiento).

\section{Procedimiento}

La primera etapa del proyecto fue buscar toda la información necesaria para trabajar con académicos que presentaran alguna de las siguientes condiciones como son: diabetes, hipertensión, obesidad y dislipidemia, con el fin de crear un programa de ejercicio físico sistematizado y adaptado a cubrir las necesidades principales, sin causar riesgo a los participantes.

La invitación se realizó por varios medios: un tríptico informativo haciéndose llegar vía correo electrónico. Un boletín informativo universitario e invitación impresa, dejándola en cada departamento académico. Se obtuvo respuesta de 28 académicos, los cuales fueron citados a una reunión donde se explicaron los objetivos de la investigación y las actividades del programa de intervención. Aceptaron 13 académicos, los cuales firmaron el consentimiento informado.

El siguiente paso: la toma de muestra sanguínea, realizada en el LACIUS. Posteriormente se citó para la toma de antropometría, apoyados por el personal del Centro de Promoción a la Salud Nutricional de la Universidad de Sonora.

Una vez realizados los análisis pertinentes, se convocó para la realización delTest de Rockport en un área deportiva de la universidad, que mide una milla su recorrido, especial para la aplicación del test de Rockport, conocida como «la milla universitaria». Se contó con el apoyo de cuatro estudiantes becados de la Licenciatura en Cultura Física y Deportes para apoyar en las mediciones del test físico, quienes acompañaron a los académicos con el fin monitorear el desempeño de la prueba y cuidar de algún síntoma de riesgo que pudiera presentarse.

Se contó con el apoyo de un médico y dos médicos pasantes de la misma institución, quienes tomaban la presión arterial y frecuencia cardiaca, antes, durante y después del entrenamiento, esto se hizo para prevenir algún problema fisiológico.

Ya analizados los resultados del test de Rockport, se 
estimó el porcentaje de la intensidad de trabajo físico para cada participante, sin exponer un riesgo a su salud, a través del método de Karvonen, llevando a cabo siempre el formato del programa establecido.

Las sesiones se llevaron a cabo en el Salón de Usos Múltiples (SUM) del Programa de Cultura Física y Deporte, adscrito al departamento de Cs. del Deporte y de la Actividad Física de la Universidad de Sonora, en un horario de 18:30 a 19:30 pm de lunes a jueves. Al inicio de la sesión, los participantes tenían un período de 10 minutos de relajación, en el cuál se les enseñaba técnicas de respiración. Posterior a esto, el personal médico registraba la frecuencia cardiaca en reposo, la saturación de oxígeno, presión arterial y en los casos necesarios la glucosa en sangre. Estos registros eran al inicio y al final de cada sesión de actividad física. Paso siguiente, se iniciaba con el calentamiento entre los cinco y 10 minutos, según la sesión de trabajo planificada para cada día de forma sistemática de acuerdo a las necesidades de los participantes, esto con el fin de obtener los mayores beneficios con los menores riesgos.

El conteo de pasos desarrollados en el test físico aplicado permitió ir graduando el aumento de forma progresiva las vueltas que se ejecutaron de lunes a miércoles durante las 13 semanas. Se tomaban retos personales de bajar el tiempo de la vuelta o aumentar la cantidad de pasos realizados conforme se iba observando la mejora de la FC. Las actividades que complementaban el desarrollo cardiorrespiratorio a parte de la caminata, se distribuyeron los jueves de cada semana, quedando de la siguiente manera: las primeras cuatro semanas correspondían a natación, con un tiempo de 30 minutos dentro del agua realizando variedad de ejercicios con intensidades de leve a moderado. De la quinta a la novena semana, se destinaron al uso de la bicicleta estacionaria, con una duración de 20 minutos entre 55 a 65\% su FCmáx. Las últimas cinco semanas se dedicaron a la actividad de baile, con un tiempo de 30 minutos, con intensidad moderada y de acuerdo al ritmo de la música, que en ocasiones llegaba a ser vigoroso por segundos, trabajando sobre una FCmáx entre 55 a 65\%. La capacidad condicional de la fuerza se llevó a cabo los lunes y miércoles, involucrando más el tren superior (brazos, espalda, abdomen) para evitar efectos negativos de cansancio o sobrecarga al tren inferior con la rutina aeróbica. Esta incluía diferentes tipos de ejercicios dirigidos a abdominales, lagartijas y semi lagartijas, bíceps, tríceps y lumbares, de seis a ocho ejercicios con ocho a 12 repeticiones. En relación a la flexibilidad, esta fue apoyado a través del método de Pilates sobre piso para principiantes. Se realizaba martes y jueves tratando de atender los ejercicios que se adecuaran a los participantes, con ocho a 12 repeticiones. Los beneficios del Pilates es que involucran la fuerza, equilibrio, concentración, flexibilidad y continua supervisión de las posturas por parte de los responsables. De lunes a jueves fue considerado el trabajo permanente de la respiración, a través del método de Vinyasa Yoga para principiantes. Estos ejercicios iban más dirigidos a la relajación muscular para aprender a sincronizar la respiración con cada movimiento corporal.

Cabe señalar que, durante las sesiones se presentaron casos en que la presión arterial sobrepasaba los valores normales y la realización de ejercicio físico incrementaba un riesgo a la salud del participante, por lo que se ajustaba la sesión para ese participante, realizando ejercicios de respiración combinado con estiramientos leves y posturas de yoga para que la frecuencia cardiaca se estabilizara. Al culminar las 13 semanas de trabajo, se realizó nuevamente el Test de Rockport, así como la toma de muestra sanguínea y las mediciones antropométricas para su análisis, interpretación y efecto en la salud.

\section{Análisis estadístico}

Para el procesamiento estadístico de los datos Correspondientes a las variables de estudio se utilizó el paquete estadístico SPSS V. 22. Se reportan medidas descriptivas numéricas para las variables cuantitativas. Para el análisis comparativo se tomaron las medidas de las variables en dos momentos, antes del programa de ejercicio físico y posterior al programa. Se utilizó la prueba de Shapiro-Wilk para comprobar el supuesto de normalidad y determinar la prueba estadística para valorar si las diferencias observadas fueron estadísticamente significativas. Dado el incumplimiento de normalidad, se utilizó la prueba no paramétrica de Wilcoxon para las variables: peso, masa magra, masa muscular, presiona arterial sistólica, diastólica y circunferencia de cintura. Para las variables: niveles de glucosa, colesterol, triglicéridos, VO2max, IMC y circunferencia de cintura, se utilizó la prueba t para muestras dependientes. Todos los análisis inferenciales se trabajarán con un nivel de significancia de .05.

\section{Confidencialidad}

Toda información personal de los académicos participantes como lo es nombre, dirección de correo electrónico, teléfono, resultados de análisis bioquímicos y resultados de los test físicos, fueron tratados de manera 
estrictamente confidencial. El investigador responsable del estudio y sus colaboradores fueron los responsables de aplicar los cuestionarios y pruebas físicas, siendo ellos los únicos con acceso a la información e identidad de los participantes en el estudio. Una vez recabados los datos necesarios para la elaboración del análisis de la información, se eliminó la evidencia de identificación personal para utilizarse los datos de manera grupal y ser presentados en reuniones y publicaciones científicas. La aplicación de las herramientas de recolección de información fue llevada a cabo por personal previamente capacitado para el cumplimiento de los objetivos del estudio.

\section{Resultados}

\section{Capacidad cardiorrespiratoria}

En el estudio participaron 10 académicos de manera voluntaria y hubo una participación activa del 100\% en las 13 semanas que duró el programa de intervención. Se logró un aumento significativo en la variable principalVO2máx de 10.41(ml/kg/min) ( $p=.002)$. De igual forma, el número de pasos tuvo un gran incremento significativo de 4046 pasos/día (Tabla 3).

En la figura 1, se reporta el comportamiento del VO2máx para cada uno de los participantes. Al inicio, el valor más bajo y el más alto fueron de $-13.285 \mathrm{ml} /$

\begin{tabular}{|c|c|c|c|}
\hline & Antes & Después & \\
\hline Variable principal & $\mathrm{M} \pm \mathrm{DE}$ & $\mathrm{M} \pm \mathrm{DE}$ & P valor \\
\hline $\mathrm{VO} 2 \max$ & $17.33 \pm 14.52$ & $27.74 \pm 11.44 .44$ & .002 \\
\hline \multicolumn{4}{|l|}{ Variables secundarias } \\
\hline Peso & $94.11 \pm 23.19$ & $91.38 \pm 22.84$ & .009 \\
\hline IMC & $35.41 \pm 5.83$ & $34.47 \pm 6.04$ & .004 \\
\hline Masa Grasa & $44.79 \pm 13.60$ & $42.39 \pm 13.53$ & .014 \\
\hline Masa Magra & $49.33 \pm 12.06$ & $48.99 \pm 12.41$ & .683 \\
\hline Masa Músculo & $23.10 \pm 6.94$ & $23.30 \pm 7.09$ & .358 \\
\hline Circunferencia cintura & $110.88 \pm 14.27$ & $106.90 \pm 14.41$ & .016 \\
\hline Glucosa & $94.69 \pm 7.98$ & $92.50 \pm 5.52$ & .345 \\
\hline Colesterol & $202.17 \pm 23.55$ & $194 \pm 10.66$ & .146 \\
\hline Triglicéridos & $131.31 \pm 22.53$ & $116.90 \pm 12.88$ & .041 \\
\hline Frecuencia Cardiaca & $143.20 \pm 17.97$ & $127.40 \pm 11.88$ & .215 \\
\hline Presión sistólica (PAS) & $122.50 \pm 12.08$ & $119 \pm 11.97$ & .406 \\
\hline Presión diastólica (PAD) & $84 \pm 9.40$ & $78 \pm 6.75$ & .058 \\
\hline Pasos & $2157.40 \pm 75.16$ & $6204.30 \pm 117.95$ & .000 \\
\hline
\end{tabular}

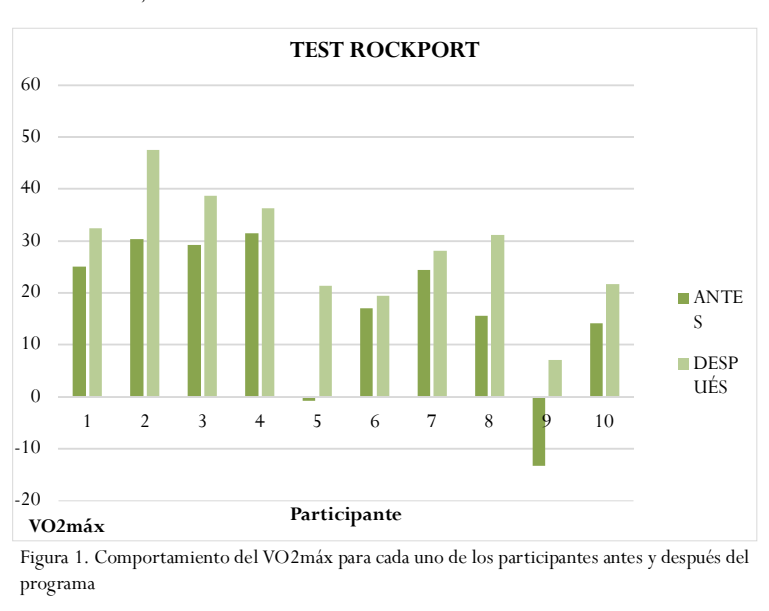

$\mathrm{kg} / \mathrm{min}$ y $31.419 \mathrm{ml} / \mathrm{kg} / \mathrm{min}$ respectivamente. Al finalizar la intervención el resultado más bajo fue de 7.075 $\mathrm{ml} / \mathrm{kg} / \mathrm{min}$ y el mayor de $47.62 \mathrm{ml} / \mathrm{kg} / \mathrm{min}$. Los diez participantes aumentaron su consumo de oxígeno y se resalta el hecho que los sujetos 5 y 9 se encontraban en el nivel más bajo, que refleja la nula condición física con la que iniciaron, después del programa su VO2máx aumentó $22.17 \mathrm{ml} / \mathrm{kg} / \mathrm{min}$ y $20.36 \mathrm{ml} / \mathrm{kg} / \mathrm{min}$ respectivamente.

\section{Medidas antropométricas}

Se logró un cambio significativo en peso $(\mathrm{p}=.009)$, IMC $(\mathrm{p}=.004)$, masa grasa $(p=.014)$ y en la circunferencia de cintura $(p=.016)$ (Tabla 3$)$.

\section{Variables metabólicas}

Los niveles de triglicéridos se redujeron significativamente $14.41 \mathrm{mg} / \mathrm{dl}(\mathrm{p}=.041)$ después del programa de intervención. Aunque los niveles de glucosa y colesterol disminuyeron al término del programa, estos no fueron estadísticamente significativos $(p>$ .05) (Tabla 3).

\section{Presión arterial}

No hubo diferencias significativas en la presión arterial sistólica ni diastólica, aunque hubo una tendencia que ésta última fuera menor después del programa de intervención $(p=.058)$ (Tabla 3).

\section{Discusión}

La presente investigación evaluó el efecto de un programa de intervención de ejercicio físico con duración de 13 semanas sobre la capacidad cardiorrespiratoria en personas con algún factor de riesgo de síndrome metabólico. La variable de respuesta fue elVO2máx. El consumo máximo de oxígeno es el indicador más válido de la funcionalidad de los aparatos cardiovascular y respiratorio que, mediante el ejercicio nos indica la capacidad que tiene el corazón, los pulmones y la sangre para transportar oxígeno a los músculos en actividad y la utilización del oxígeno por los músculos durante el ejercicio. El programa logra un aumento significativo de $10.41 \mathrm{ml} / \mathrm{kg} / \mathrm{min}$ en esta variable. Estudios previos han reportado valores cercanos a los resultados en esta investigación, aunque con diferente intervención y duración, como es el caso del estudio de Hurst, Weston \& Weston (2018), quienes aplicaron un programa de intervención de alta intensidad de entrenamiento físico a adultos sanos mayores de 50 años durante 24 sesiones, 
logrando un cambio significativo en elVO2máx de 11.5 $\mathrm{ml} / \mathrm{kg} / \mathrm{min}$. Otros estudios en poblaciones similares no han tenido una respuesta muy favorable como el de Lunt, Draper, Marshall, Logan, Hamlin, Shearman et al. (2014), en donde se compara la capacidad cardiorrespiratoria de tres grupos de personas con sobrepeso que participan en diferentes niveles de entrenamiento físico: caminata con baja intensidad, otro con entrenamiento aeróbico y el último con intensidad volitiva máxima encontrando que el entrenamiento con intervalo aeróbico produjo un mayor cambio en el VO2máx. Los autores concluyen que los sujetos con sobrepeso que se someten a entrenamiento a intervalos de alta intensidad en un entorno real mejora el estado cardiorrespiratorio, sin embrago, hay resultados contradictorios, en donde declaran que el VO2máx mejora si el sujeto se somete a un programa de entrenamiento continuo de intensidad moderada comparado con aquellos con intervalos de alta intensidad (Ciolac, Bocchi, Bortolotto, Carvalho, Greve \& Guimarães, 2010; Fisher, Brown, Bohan, Alcorn, Noles, Winwood et al., 2015). La adaptabilidad cardíaca y vascular al acondicionamiento, se observa con el resultado de la disminución de la frecuencia cardíaca promedio durante la realización de ejercicios aeróbicos, logrando enfatizar en la adquisición de resistencia física por parte de los sujetos de estudio.

Diferentes estudios han demostrado como un programa de actividad física de moderada a vigorosa causa un aumento significativo en la masa libre de grasa y disminución en la masa grasa (Macpherson, Hazell, Olver, Paterson \& Lemon, 2011) así como una disminución significativa en la masa grasa y abdominal en mujeres con sobrepeso (Gillen, Percival, Ludzki, Tarnopolsky \& Gibala, 2013). En este estudio, se observa un aumento significativo en la masa corporal, como producto de la disminución de la masa grasa la cual fue de $2.4 \mathrm{~kg}$. Cuando hay un aumento del consumo máximo de oxígeno, por lo general hay un gasto calórico que repercute positivamente en la masa grasa y beneficia la masa muscular y aunque en este estudio no aumentó significativamente ésta, el aumento en el VO2máx permite que disminuya el esfuerzo del corazón y se colabora para mejorar la calidad de vida. Estos resultados son congruentes con otros estudios en donde no se han observado cambios importantes después de una intervención en algunas de estas variables (Ciolac et al., 2010; Galaviz, Trejo, Borbón, Alarcón, Pineda, Arrayales et al., 2021).

En cuanto al número de pasos, los participantes ini- ciaron con un promedio 2157 pasos por día. Una persona se considera sedentaria si realiza menos de 5000 pasos/ día y poco activos si realiza entre 5000 y 7499 pasos/día (Tudor \& Basset, 2004). Los participantes lograron alcanzar un promedio de más de 6000 pasos/día. Considerando que los participantes no eran sujetos sanos y que no realizaban ninguna actividad física, estos al final del programa fueron clasificados como «poco activos». Implementar el hábito de la caminata es una manera muy efectiva de incrementar los niveles de actividad física (Tudor-Locke et al., 2004). En los últimos años la caminata ha alcanzado cierta popularidad, sin embargo, existe evidencia de que la gente no camina con la suficiente frecuencia como para cumplir con las recomendaciones de salud pública (Rafferty, Reeves, McGee \& Pivarnik, 2002).

Unido a estos beneficios la disminución de la circunferencia de cintura es notoria, los participantes lograron reducir su perímetro abdominal en $3.98 \mathrm{~cm}$. Es importante señalar que la circunferencia de cintura según la Federación Internacional del Diabetes, es un predictor de riesgo cardiovascular, la adiposidad central es parte de los componentes del síndrome metabólico. Así que una circunferencia de grandes dimensiones estaría dentro del diagnóstico del síndrome metabólico. Afortunadamente existen alternativas a bajo costo que benefician enormemente la salud, entre ellos el cambio de hábitos en los estilos de vida, reducción del peso y aumentar el tiempo de actividad física, pudiendo ser este programa un ejemplo para proponerse una rutina de caminata a través de los pasos realizados. Los cambios observados en este estudio han sido similares a los reportados en otras investigaciones, sobre todo en la circunferencia cintura (Lunt et al., 2014).

También se ha demostrado que el ejercicio físico aumenta el colesterol HDL y reduce los triglicéridos y el colesterol de lipoproteínas de baja densidad (LDL). El estudio HERITAGE de León, Rice, Mandel, Despre's, Bergeron, Gagnon et al. (2000), en donde se estudiaron los efectos de 20 semanas de ejercicio supervisado en sujetos sanos y sedentario reportó un aumento de 1.4 mg por $100 \mathrm{ml}$ en los niveles de colesterol HDL y una disminución de $0.6 \mathrm{mg}$ por $100 \mathrm{ml} \mathrm{y} 4.4 \mathrm{mg}$ por $100 \mathrm{ml}$ en los valores de triglicéridos y colesterol LDL respectivamente. En los datos obtenidos se observa una disminución significativa en los niveles de triglicéridos de $14.41 \mathrm{mg}$ por $100 \mathrm{ml}$. En cuanto al colesterol LDL y niveles de glucosa, estos muestran una reducción de 8.17 y $2.19 \mathrm{mg}$ por $100 \mathrm{ml}$ respectivamente, colocando a dos participantes en la categoría de bajo riego, aunque 
estas disminuciones no fueron estadísticamente significativas. Estos resultados coinciden con otros estudios, en donde el ejercicio no ha causada un efecto significativo en estos lípidos. Ciolac et al. (2010), aplicaron un programa de entrenamiento aeróbico en intervalos de alta intensidad y mostraron efectos positivos en estas variables, mientras que el grupo sometido a ejercicio moderado no detectaron una diferencia significativa. Estos resultados contradictorios en algunos estudios pueden deberse al tipo de programa de actividad física que se cometen los sujetos, así como las características de los mismos. Tal es el caso del reportado en OchoaMartínez, Hall-López, Piña, Zarate \& Teixeira (2019), donde se midió el efecto de un entrenamiento de ejercicio en el agua sobre los componentes del síndrome metabólico en mujeres mayores de 60 años aparentemente sanas. Los autores solo encontraron un cambio significativo en los niveles de triglicérido.

Sobre la presión arterial no se observan una diferencia estadísticamente significativa $(p>.05)$. Estos resultados pueden deberse al tamaño de la muestra y al diseño experimental utilizado. Desde una perspectiva clínica, una disminución de $2 \mathrm{mmHg}$ en la presión arterial sistólica es probable que la mortalidad asociada con accidentes cerebrovasculares se reduzca en un 6\% y de enfermedad coronaria en un 4\% (Chobanian, Bakris, Black, Cushman, Green, Izzo et al., 2003). Por lo que, la reducción de $3.50 \mathrm{mmHg}$ en la PAS después del programa de intervención evidencia la importancia de la actividad física. Por otro lado, esta reducción y los 6 mmHg de disminución en la PAD tuvo un efecto cualitativo, ya que se observó una reducción del 50\% en el porcentaje de personas con niveles crónicos de la presión arterial y los sujetos con niveles fronterizo normalizaron su presión arterial después del programa de intervención. Los resultados favorables se lograron más en las mujeres, contrario a lo reportado por otros estudios, que declaran que los hombres logran mayores reducciones en la presión arterial sistólica que las mujeres (Christou, Jones, Jordan, Diedrich, Robertson \& Seals, 2005; Carpio-Rivera, Moncada-Jiménez, SalazarRojas \& Solera-Herrera, 2016). Otro dato que se encontró fue que en los participantes de menor edad se produjo una mayor reducción de la PAS y PAD. Estos resultados apoyan lo declarado por otros investigadores que afirman que cuanto mayor sea la edad de los participantes, menor será la reducción de la PAD (Canuto, Nogueira, Cunha, Ferreira, Pinto, Costa et al., 2011; Carpio-Rivera et al., 2016). Por otro lado, existe mucha evidencia que relaciona la prevalencia de hipertensión con obesidad (Rahmouni, Correia, Haynes, Mark, 2005; Hamer, 2006; Miyai, Shiozaki,Yabu, Utsumi, Morioka, Miyashita et al., 2013). En este estudio, fue evidente la asociación entre el IMC y la PAS, en donde se observa que los sujetos con menor IMC disminuyeron más su presión arterial sistólica. Estos resultados son importantes, dado que más del 70\% de la población adulta en México presenta sobrepeso y obesidad (Rivera, Colchero, Fuentes, González de Cosío, Aguilar, Hernández, et al., 2018) y esto puede justificar el hecho de que también es el país que registra la tasa de prevalencia más alta de morbilidad y mortalidad a causa de la hipertensión arterial (Baglietto-Hernández, Mateos-Bear, Nava-Sánchez, Rodríguez-García \& Rodríguez-Weber, 2020). Este estudio tuvo una duración de 13 semanas y se observaron cambios importantes para la salud de los participantes.

Una de las limitaciones del presente estudio es el tamaño de muestra y la falta de un grupo de control; por lo que existe la posibilidad de que los cambios observado en el VO2máx puedan deberse al efecto de la repetición y aunque este estudio ha demostrado que un programa de actividad física puede provocar grandes beneficios a sujetos con problemas de sobrepeso u obesidad, se debe de tener en cuenta que se trata de un estudio piloto, por lo que se recomienda seguir explorando con una muestra más grande y con otra metodología.

\section{Conclusiones}

Si bien, es bastante conocido que el ejercicio físico tiene muchos beneficios para la salud de los individuos, hace falta mayor promoción en las instituciones de educación de los diferentes niveles de impulsar la práctica constante del ejercicio en su población. Este trabajo se focalizó en los padecimientos de síndrome metabólico, encontrando mejorías principalmente en la capacidad cardiorrespiratoria, así como en parámetros antropométricos como fue el peso $(\mathrm{kg})$, la masa grasa y la circunferencia de cintura. Cabe mencionar que no se llevó un régimen alimenticio, sólo se compartieron recomendaciones.

Es importante destacar que no hay investigaciones previas para este tipo de proyecto, por lo que se sugiere seguir investigando al respecto y documentar los hallazgos. También se recomienda que las instituciones adopten programas de ejercicio físico para su personal, esto es con el fin de combatir o prevenir enfermedades crónicas no transmisibles y mejorar la calidad de vida 
de sus empleados.

Se llega a la conclusión que, difícilmente se puede incidir en un ser humano para beneficiar su salud si no es por conciencia propia. Desafortunadamente la falta de tiempo es un factor que limita las actividades para mejorar la salud. En un mundo cibernético que cansa y que envuelve las actividades propias de académicos e investigadores, quizá buscando una meta profesional o una meta personal, se olvida de lo principal que es la meta de la salud.

Se pudo observar que decenas de académicos que respondieron al llamado, que comunicaban su estado de salud: obesidad, sobrepeso, diabetes, hipertensión, como también expresaban «que les encantaría participar», llegado el tiempo, solo se sumaron trece y quedando diez, optando por justificarse con barreras como: las clases, los horarios, las investigaciones, las distancias, entre otras que no les permitían participar.

Aun así, el programa de intervención propuesto fue muy bien recibido. Parece sencillo dar una caminata, pero conlleva a una reeducación en el plano motor, corregir las malas posturas que forman parte de malos hábitos, una adecuada respiración con ritmo adecuado, un braceo preciso para no lesionar articulaciones o el impacto del tren inferior al piso. La ropa adecuada, la hidratación, la intensidad que se debe realizar para obtener resultados, y la parte volitiva del participante, son parte esencial para que la caminata brinde los resultados en salud esperados. Es parte de lo que aprendieron los docentes participantes.

Los resultados obtenidos es este estudio piloto indican que este tipo de programas de bajo costo, fáciles de aplicar, con un proceso metodológico sistematizado y sobre todo con un equipo multidisciplinario involucrado pueden lograr resultados favorables para la salud.

Fue un gran aprendizaje y punto de reflexión las barreras que el ser humano expone ante su salud, y como otros aprovechan las oportunidades para mejorarla. Se puede concluir que quienes lograron este cambio continuaran mejorando lo más preciado que tienen: la salud.

\section{Referencias}

Abellán, J., Sainz de Baranda, Ortín, E.J., Saucedo, P., Gómez, P., \& Leal, M. (2014). Guía para la prescripción del ejercicio físico en pacientes con riesgo cardiovascular. Recuperado de http://www.seh-lelha.org/pdf/ guiaejerciciorcv.pdf

Alvarez-Pitti, J., Casajús-Mallén, J.A., Leis-Trabazo, R., Lucía, A., López de Lara, D., Moreno-Aznar, L.A., \& Rodríguez-
Martínez, G. (2020). Exercise as medicine in chronic diseases during childhood and adolescence. Anales de Pediatria, 92(3), 173.e1-173.e8. Recuperado de https:/ / doi.org/10.1016/j.anpedi.2020.01.010

Baglietto-Hernández, J.M., Mateos-Bear,A., Nava-Sánchezm J.P., Rodríguez-García, \& P., Rodríguez-Weber, F. (2020). Nivel de conocimiento en hipertensión arterial en pacientes con esta enfermedad de la Ciudad de México. Med Int Méx., 36(1), 1-14. Recuperado de https://doi.org/ 10.24245/mim. v36i1.2844

Canuto, P.M., Nogueira, I.D., Cunha, E.S., Ferreira, G.M., Pinto, K.M., Costa, F.A., ... Silva, P.A. (2011). Influence of resistance training performed at different intensities and same work volume over BP of elderly hypertensive female patients. Rev Bras Med Esporte, 17(4), 246-9. Recuperado de https://www.scielo.br/pdf/rbme/v17n4/ en_v17n4a06.pdf

Carpio-Rivera, E., Moncada-Jiménez, J., Salazar-Rojas,W., \& Solera-Herrera,A. (2016). Efectos agudos del ejercicio sobre la presión arterial: una investigación metaanalítica. Arquivos brasileiros de cardiologia, 106 (5), 422433. Recuperado de https://doi.org/10.5935/ abc. 20160064

Castañeda-Lechuga, C., Macias-Ruvalcaba, S., GallegosSánchez, J., \& Villareal-Angeles, M. (2019). Mejora de constructos físicos en adultos mayores de la zona norte de México (Improvement of physical fitness components in older adults from northern México). Retos, 37, 258-263. https://doi.org/10.47197/retos.v37i37.62258

Cerecero,P.,Hernández, B.,Aguirre, D., Valdés, R., \& Huitrón, G. (2009). Estilos de vida asociados al riesgo cardiovascular global en trabajadores universitarios del Estado de México. Salud Pública de México, 51(6), 465-473. Recuperado de http://www.scielo.org.mx/

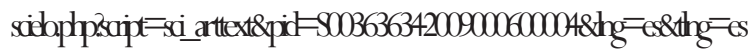
ChávezValenzuela, M., Bautista Jacobo,A., García Fernàndez, D., Fuentes Vega, M., Ogarrio Perkins, C., Montaño Del Cid, E., \& Hoyos Ruíz, G. (2018). APPLICATION OF AN INTERVENTION PROGRAMFORTHE BENEFIT OF PHYSICAL AND EMOTIONAL HEALTH IN ELDERLY ADULTS OF FEMALE SEX OF HERMOSILLO, SONORA. MHSalud: Revista En Ciencias Del Movimiento HumanoY Salud, 15(1), 1-15.https:// doi.org/10.15359/mhs.15-1.4

Chobanian, A.V., Bakris, G.L., Black, H.R., Cushman,W.C., Green, L.A., Izzo, J.L Jr., \& Jones, D.W. (2003). The Seventh Report of the Joint National Committee on Prevention, Detection, Evaluation, andTreatment of High Blood Pressure: the JNC 7 report.JAMA, 289(19),256072. Recuperado de https://doi.org/10.1001/ jama.289.19.2560

Christou, D.D., Jones, P.P., Jordan, J., Diedrich,A., Robertson, 
D., \& Seals, D.R. (2005). Women have lower tonic autonomic support of arterial blood pressure and less effective baroreflex buffering than men. Circulation, 111(4), 494-8. Recuperado de https://doi.org/10.1161/ 01.cir.0000153864.24034.a6

Ciolac, E.G., Bocchi, E.A., Bortolotto, L.A., Carvalho, V.O., Greve, J.M., \& Guimarães, G.V. (2010). Effects of highintensity aerobic interval training vs. moderate exercise on hemodynamic, metabolic and neuro-humoral abnormalities of young normotensive women at high familial risk for hypertension. Hypertens Res., 33(8), 83643. Recuperado de https://doi.org/10.1038/ hr. 2010.72

Encuesta Nacional de Salud y Nutrición de Medio Camino (2016). Informe Final de Resultados. Instituto Nacional de Salud Pública. México, Recuperado de https:/ / www.gob.mx/salud/documentos/encuesta-nacionalde-salud-y-nutricion-de-medio-camino-2016

Federación Internacional del Diabetes (2006). Recuperado de https://www.fundaciondiabetes.org/diamundial/ 350/2006-diabetes-y-las-personas-desfavorecidas-yvulnerables

Fisher, G., Brown,A.W., Bohan, M.M.,Alcorn,A., Noles, C., Winwood, L., ... Resuehr, H. (2015). High Intensity Interval- vs Moderate Intensity-Training for Improving Cardiometabolic Health in Overweight or Obese Males: A Randomized ControlledTrial. PLoS ONE, 10(10), 1-15. Recuperado de https://doi.org/10.1371/ journal.pone.0138853

Ford, E.S. (2005). Prevalence of the metabolic syndrome defined by the International Diabetes Federation among adults in the U.S. Diabetes Care, 28(11), 2745-2749. Recuperado de https://pubmed.ncbi.nlm.nih.gov/ $16249550 /$

Gillen, J.B., Percival, M.E., Ludzki,A.,Tarnopolsky, M.A., \& Gibala, M.J. (2013). Interval training in the fed or fasted state improves body composition and muscle oxidative capacity in overweight women. Obesity (Silver Spring), 21(11), 2249-55. Recuperado de https://doi.org/ 10.1002 /oby. 20379

Galaviz Berelleza, R., Trejo Trejo, M., Borbón Román, J., Alarcón Meza, E., Pineda Espejel, H., Arrayales Millan, E., Robles Hernández, G., \& Cutti Riveros, L. (2020). Efecto de un programa de entrenamiento de fuerza sobre IGF-1 en adultos mayores con obesidad e hipertensión controlada (Effect of a strength training program on IGF1 in older adults with obesity and controlled hypertension). Retos, 39, 253-256. https://doi.org/ $10.47197 /$ retos.v0i39.74723

García González, A., \& Froment, F. (2017). Beneficios de la actividad física sobre la autoestima y la calidad de vida de personas mayores (Benefits of physical activity on self- esteem and quality of life of older people). Retos, 33, 3-9. https: / / doi.org/10.47197/retos.v0i33.50969

Guillen Pereira, L., Bueno Fernandez, E., Gutierrez Cruz, M., \& Guerra Santiesteban, J. (2017). Programa de actividad física y su incidencia en la depresión y bienestar subjetivo de adultos mayores (Impact of a physical activity program on older adults' depression and subjective wellbeing). Retos, 33, 14-19. https://doi.org/10.47197/ retos.v0i33.49638

Gutiérrez, J., Rivera, J., Shamah,T., Oropeza, C., \& Hernández, M. (2012). Encuesta Nacional de Salud y Nutrición 2012. Resultados Nacionales. Instituto Nacional de Salud Pública, Recuperado dehttps: / / ensanut.insp.mx/encuestas / ensanut 2012 / doctos / informes / ENSANUT2012ResultadosNacionales.pdf

Hamer, M. (2006). The anti-hypertensive effects of exercise: integrating acute and chronic mechanisms. Sports Med., 36(2), 109-16. Recuperado de https://doi.org/ 10.2165/00007256-200636020-00002

Hernández, R., Fernández, C., \& Baptista, M. (2010). Metodología de la Investigación. $5^{\text {th }}$. ed. McGraw Hill, México.

Heyward, V. (2008). Advanced Fitness Assessment and Exercise Prescription. $5^{\text {th }}$. ed. Panamericana, España.

Hurst, C.,Weston, K.L., \&Weston, M. (2018). The effect of 12 weeks of combined upper- and lower-body highintensity interval training on muscular and cardiorespiratory fitness in older adults. Aging Clin Exp Res., 31(5),661-671. Recuperado de https: / / doi.org/ 10.1007/s40520-018-1015-9

Kline, G.M., Porcari, J.P., Hintermeister, R., Freedson, P.S., Ward, A., McCarron, R.F., ... Rippe, J.M. (1987). Estimation of $\mathrm{VO}_{2}$ max from a 1-mile track walk, gender, ege, and body weight. Medicine and Science in Sports and Exercise, 19, 253-259.

Leon, A.S., Rice, T., Mandel, S., Despre's, J.P., Bergeron, J., Gagnon, J., Rao, D.C., Skinner, J.S., Wilmore, J.H., \& Bouchard, C. (2000). Blood lipid response to 20 weeks of supervised exercise in a large biracial population: the HERITAGE Family Study. Metabolism, 49(4), 513-520. Recuperado de https://pubmed.ncbi.nlm.nih.gov/ 10778878/

Lunt, H., Draper, N., Marshall, H.C., Logan, F.J., Hamlin, M.J., Shearman, J., . . F Frampton, Ch.M.A. (2014). High Intensity Interval Training in a Real World Setting: A Randomized Controlled Feasibility Study in Overweight Inactive Adults, Measuring Change in Maximal Oxygen Uptake. PLoS ONE, 9(1), 1-11. Recuperado de https: / / doi.org/10.1371/journal.pone.0083256

Macpherson, R.E., Hazell,T.J., Olver,T.D., Paterson, D.H., \& Lemon, P.W.(2011). Run sprint interval training improves aerobic performance but not maximal cardiac output. Med Sci Sports Exerc., 43(1), 115-22. Recuperado de https: / / 
doi.org/10.1249/MSS.0b013e3181e5eacd

Miyai, N., Shiozaki, M., Yabu, M., Utsumi, M., Morioka, I., Miyashita, K, \& Arita, M. (2013). Increased mean arterial pressure response to dynamic exercise in normotensive subjects with multiple metabolic risk factors. Hypertens Res., 36(6), 534-9. Recuperado de https:// www.nature.com/articles/hr2012215

National Cholesterol Education Program. (2001).Third report of the National Cholesterol Education Program (NCEP) expert panel on: Detection, evaluation and treatment of high blood cholesterol in adults (Adult treatment panel Ill). National Heart, Lung and Blood Institute. Recuperado de https://www.nhlbi.nih.gov/files/docs / resources/heart/atp-3-cholesterol-full-report.pdf

Ochoa-Martinez, P.Y., Hall-López, J.A., Piña, D., Zárate, D.A., \&Teixeira,A.M. (2019). Effects of three months of waterbased exercise training on metabolic syndrome components in older women. Retos, 35, 181-184.

Organización Mundial de la Salud. (2013). Información general sobre la Hipertensión en el mundo. Una enfermedad que mata en silencio, una crisis de salud pública mundial. Organización Mundial de la Salud. Suiza, p. 40. Recuperado de https: / /apps.who.int/iris/bitstream/handle/ $\begin{array}{lllllllllll}1 & 0 & 6 & 6 & 5 & / & 8 & 7 & 6 & 7 & 9\end{array}$ WHDDCOLHD_ D132

Organización Panamericana de la Salud (OPS). (2021). Recuperado de https: / /www.paho.org/es/heartsamericas/hearts-americas-medicion-presion-arterial

Quintana-Zavala, M.O.,Vílchez-Barboza,V., Figueroa-Ibarra, C., García-Puga, J.A., Tinajero-González, R.M., \& SalazarRuibal, R.E. (2018). Efecto De Una Consejería De Enfermería Sobre Factores De Riesgo Cardiovascular Modificables Dirigida a Empleados. Effect of a Nursing Counseling on Modifiable Cardiovascular Risk Factors AddressedTo Employees. Dirección De Correspondencia. Revista Mexicana de Enfermería Cardiológica, 26(1), 20 26. Recuperado de www.revistamexicanadeenfermeriacardiologica.com.mx

Rafferty, A.P., Reeves, M.J., McGee, H. B., \& Pivarnik, J.M. (2002). Physical activity patterns among walkers and compliance with public health recommendations. Med. Sci. Sports Exerc., 34, 1255-1261.

Rahmouni, K., Correia, M.L., Haynes, W.G., Mark, A.L. (2005). Obesity-associated hypertension: new insights into mechanisms. Hypertension, 45(1), 9-14. Recuperado de https: / / www.ahajournals.org/doi / 10.1161/ 01.HYP.0000151325.83008.b4

Ramírez, M.A., Rosety, J.M., Marcos-Becerro, J., Rosety, I., Ordoñez, F., Rosety-Rodríguez, M., Rodríguez-Pareja, M., \& Rosety, M.A. (2012). El ejercicio y el síndrome metabólico. Rev Med Urug, 28(4), 309-316.

Rivera, J.A., Colchero, M.A., Fuentes, M.L., González de
Cosío, T., Aguilar, C., Hernández, G., \& Barquera, S. (eds.) (2018). La obesidad en México. Estado de la política pública y recomendaciones para su prevención y control. Cuernavaca: Instituto Nacional de Salud Pública, México. Recuperado de https: / / www.insp.mx/avisos/4884-laobesidad-mexico.html

Sociedad Española para el estudio de la Obesidad (2000). Recuperado de https://www.seedo.es/images/site/ d o c u m e n t a c i o n C o n s e n s o / Consenso_SEEDO_2000.pdf

Tojal, L., Alonso-Gómez, A., Alberich, S., Wärnberg, J., Sorto, C., Portillo, M. P., Schröder, H., Salas-Salvadó, J., \& Arós, F. (2020). Association between maximal oxygen consumption and physical activity and sedentary lifestyle in metabolic syndrome. Usefulness of questionnaires. Revista Española de Cardiología (English Edition), 73(2), 145152. Recuperado de https://doi.org/10.1016/ j.rec.2018.08.027

Tudor-Locke, C., Ham, S.A., Macera, C.A., Ainsworth, B.E., Kirtland, KA., Reis, J.O., \& Kimsey, C.D. (2004). Descriptive Epidemiology of pedometer-determined physical activity. Medicine and Science in Sport and exercise, 36, 1567-1573. Recuperado de http:// journals.lww.com/acsm-msse/pages/default.aspx

Tudor-Locke, C., \& Bassett, D. R. (2004). How many steps/ day are enough? Sports medicine, 34, 1-8. Recuperado de doi: 10.2165/00007256-200434010-00001

Villarreal Ángeles, M., Moncada Jiménez, J., Gallegos Sánchez, J. J., \& Ruiz-Juan, F. (2016). El efecto de un programa de ejercicios basado en Pilates sobre el estado de ánimo en adultos mayores Mexicanos (Effects of a Pilates-based exercise program on mood states in older adults in Mexico). Retos, 30, 106-109. https://doi.org/ 10.47197/retos.v0i30.49543

Villareal, D.T., Miller, B. V., Banks, M., Fontana, L., Sinacore, D.R., \& Klein, S. (2006). Effect of lifestyle intervention on metabolic coronary heart disease risk factors in obese older adults. Am J Clin Nutr., 84(6), 1317-1323. Recuperado dehttps: / / pubmed.ncbi.nlm.nih.gov/17158411/ Yassine, H.N., Marchetti, C.M., Krishnan, R.K.,Vrobel,T.R., Gonzalez, F., \& Kirwan, J.P. (2009). Effects of exercise and caloric restriction on insulin resistance and cardiometabolic risk factors in older obese adults-A randomized clinical trial. J Gerontol A Biol Sci Med Sci., 64A(1), 90-95. Recuperado de https:// www.ncbi.nlm.nih.gov/pmc/articles/PMC2691195/ 